\title{
A Markov Chain Model for Evaluating the Effectiveness of Randomized Surveillance Procedures
}

Thomas A. Edmunds

January, 1994

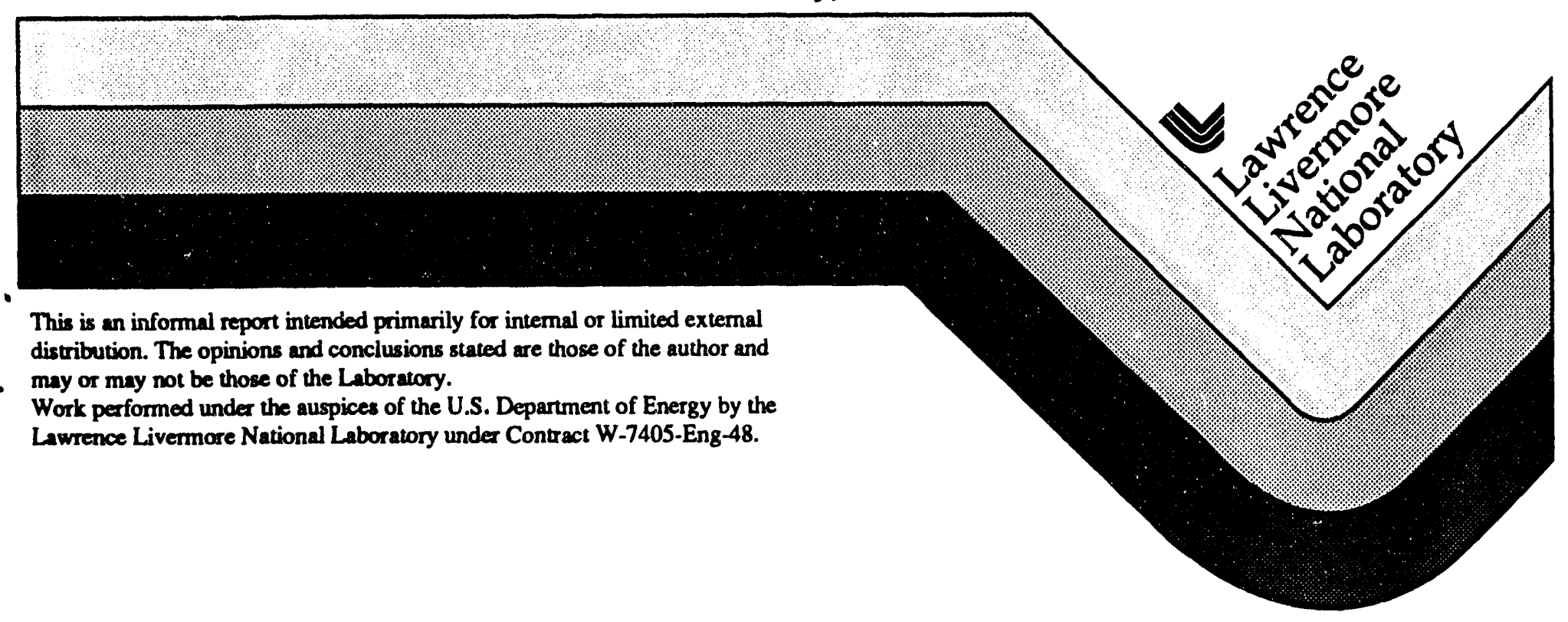




\section{DISCLAIMER}

This document was prepared as an account of work sponsored by an agency of the United States Government. Neither the United States Government nor the University of California nor any of their employees, makes any warranty, expressed or implied, or assumes any legal liability or responsibility for the accuracy, completeness, or usefulness of any information, apparatus, product, or process disclosed, or represents that its use would not infringe privately owned rights. Reference herein to any specific commercial products, process, or service by trade name, trademark, manufacturer, or otherwise, does not necessarily constitute or imply its endorsement, recommendation, or favoring by the United States Government or the University of California. The views and opinions of authors expressed herein do not necessarily state or reflect those of the United States Government or the Universiiy of California, and shall not be used for advertising or product endorsement purposes.

This report has been reproduced directly from the best available copy.

Available to DOE and DOE contractors from the

Orfice of Scientific and Technical Information

P.O. Box 62, Oak Ridge, TN 37831

Prices available from (615) 576-8401, FTS 626-8401

Available to the public from the

National Technical Information Service

U.S. Department of Commerce 5285 Port Road Royal Rd., Springfield, VA 22161 


\section{A Markov Chain Model for Evaluating the Effectiveness of Randomized Surveillance Procedures}

Thomas A. Edmunds

January 1994

Fission Energy and Systems Safety Program

Lawrence Livermore National Laboratory

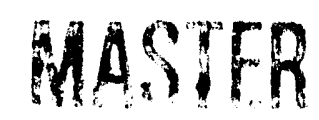




\begin{abstract}
A Markov Chain Model has been developed to evaluate the effectiveness of randomized surveillance procedures. The model is applicable for surveillance systems that monitor a collection of assets by randomly selecting and inspecting the assets. The model provides an estimate of the detection probability as a function of the amount of time that an adversary would require to steal or sabotage the asset. An interactive computer code has been written to perform the necessary computations.
\end{abstract}




\subsection{Introduction}

Consider a collection of assets that must be protected from theft, sabotage, or harm by an adversary. Assets could include critical pieces of process equipment that might be sabotaged, valuable items that might be stolen, or personnel that could be injured or killed. Assume that a surveillance system has been implemented to protect these assets from adversary activities. Surveillance systems could inciude guards pacrolling an area containing the assets, closed circuit IV (CCTV) systems, manual or electronic audits of account balances, or communications with personnel. The surveillance system monitors the assets by selecting individual assets and inspecting them for evidence of unauthorized activity by an adversary (e.g., a guard would check the first asset, then check the second asset, etc.).

The model assumes that an adversary requires a finite amount of time to implement the theft, sabotage, or assault and is susceptible to detection by a elements of the surveillance system during that time period. For example, a guard patrolling an area containing a number of valuable assets may check a particular asset during the time frame in which a theft is being attempted, observe the adversary's activities, and stop him.

If surveillance capability is insufficient to monitor simultaneously all of the assets requiring protection, the assets could be monitored by sequental inspection. However, if the assets were inspected in accordance with a fixed pattern then an adversary could observe the inspection pattern and exploit it. Therefore, in many cases it is important to implement a randomized asset inspection pattem so that an adversary would not know which asset will be inspected next.

The purpose of this paper is to describe a methodology developed to estimate the probability that a surveillance system executing a randomized inspection pattem of a collection of assets will detect an unauthorized act by an adversary. The model can be used to determine whether a particular surveillance system meets design requirements or to do cost-benefit analyses of potential surveillance system modifications. The model has been used to evaluate the effectiveness of surveillance systems at a U. S. Department of Energy facility.

The next section describes randomized surveillance procedures and their mathematical representation in the model. Section 3.0 describes the Markov Chain Model of the surveillance procedures and adversary activities and provides an example calculation. Section 4.0 describes the computer implementation of the model. The last section provides a summary and conclusions.

\subsection{Randomized Surveillance Procedures}

A sequential randomized surveillance procedure may or may not detect the activities of an adversary. The probability of detection depends upon the average frequency with which the particular asset being compromised is inspected, as well as the amount of time required 
for the adversary to complete the unauthorized act. The probability of detection increases if the inspection frequency is increased or if the adversary's task time increases.

To facilitate modeling this process and quantifying these impacts, it is useful to develop a mathematical representation of the current state of the surveillance system and changes in the state of the system. For example, the system state may be specified by identifying the asset that a guard is currently inspecting and the asset that the adversary is attempting to compromise. All possible states of the surveillance system can be represented in terms of a network diagram. Changes in the state of the surveillance system can be viewed in terms of movement in the network at discrete time steps. These network models are described below for two general types of geometries: regular and irregular geometries.

\subsection{Network for Regular Geometries}

Assets being protected may be configured in an array or lattice, as depicted in the network diagram below in Figure 2.1. The circles in the diagram represent assets to be protected

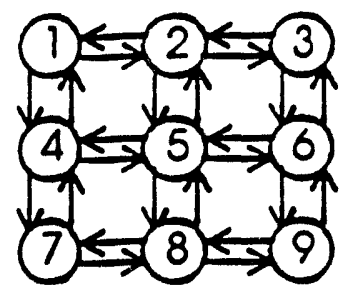

Figure 2.1 Regular array of assets to be protected

and will be referred to as nodes. The arrows connecting the nodes represent sequential choices of assets for inspection and will be referred to as arcs. The surveillance system selects one of the nine nodes for inspection, and then selects an adjacent node for the next inspection. A randomized inspection pattern implies that each adjacent node has some probability of being selected. For example, if node 1 has just been inspected, one might specify the surveillance system so that there is a 0.5 probability that node 2 will be inspected next and a 0.5 probability that node 4 will be inspected next. These probabilities will be referred to as transition probabilities, where $\mathrm{p}_{\mathrm{ij}}$ is used to indicate the transition probability from state $i$ to state $j$. A randomized surveillance system would correspond to an assignment of transition probability values to all of the ares in the network. The collection of transition probabilities are frequently presented as a matrix, called a state transition matrix, designated $\mathbf{P}$.

The model assumes that the time required to implement the next inspection does not depend upon either the node currently being inspected or the node that has just been inspected. Thus, a discrete time step, $t$, may be defined for the inspection model. The surveillance system can be viewed as moving from one node in the network to an adjacent node during each time step $t$.

As an example, the nodes shown in Figure 2.1 could represent the physical locations of pieces of process equipment that must be protected against sabotage. The surveillance system could consist of a guard patrolling the area. The guard would move from one 
piece of equipment to the next. If the pieces of equipment are evenly spaced, the amount of time required to move from one piece of equipment to the next would not depend upon the origin or destination of the guard. The time step would be the amount of time required for the guard to move from one piece of equipment to the next and inspect the equipment. The guard's trajectory would be governed by the probability values assigned to the network and would be random.

\subsection{Network for Irregular Geometries}

The network model described above can be expanded to address cases where assets do not appear in a regular array. For example, if assets are naturally partitioned into two groups, the following network model might be appropriate.

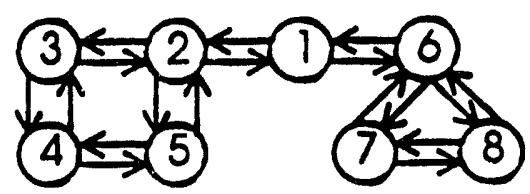

Figure 2.2 Two groups of assets

In the example shown above, nodes 2 through 5 form a first group of assets, and nodes 6 through 8 form a second group. If the surveillance system starts at node 1 , there is some probability of selecting each of the two groups for inspection. After one group has been selected, assets within that group are inspected until the system returns to node 1 and there is a possibility of selecting the second group. Note that in this configuration, node 6 would tend to be inspected more often than nodes 7 and 8 if the transition protabilities between nodes 6,7 , and 8 were equal. The inspection rate for node 6 could be reduced if the transition probabilities $\mathrm{P}_{67}$ and $\mathrm{P}_{68}$ were assigned values greater than $\mathrm{P}_{76}$ and $\mathrm{P}_{86}$

When building a network model of a randomized surveillance process, it is important to structure the network so that each node-to-node transition requires the same amount of time. In some cases it may be necessary to add nodes and ares to properiy represent the time required to inspect the next asset.

For example, consider a surveillance system in which the choice of the next asset for inspection is unrestricted (i.e. any one of the assets could be selected for the next inspection). The star-shaped network shown below could be used to represent such a system. In the figure, node 1 is a dummy node used for modeling purposes and does not represent an asset in this example. The time step (i.e. the time required to move from one node to an adjacent node) is defined as one half of the time required to complete an inspection and select the next asset for inspection. Thus, each of the six assets represented by nodes 2 through 7 have the same probability of being selected for inspection every two time steps. 


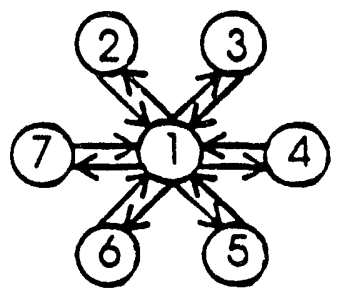

Figure 2.3 Star nerwork for unrestricted inspection choice

\subsection{Asset targeted by adversary}

An adversary may target any one of the assets being protected. He will be detected only if the surveillance system happens to select the targeted asset for inspection during the time period in which the asset is being compromised. This detection process can be represented in the network by introducing an absorbing state. The absorbing state represents capture of the adversary. If the system ever makes a transition to an absorbing state, it stays in that state.

As an example, consider the surveillance system depicted below, in which the adversary is assumed to have targeted the asset at node 3 . The numbers next to the arcs in the figure

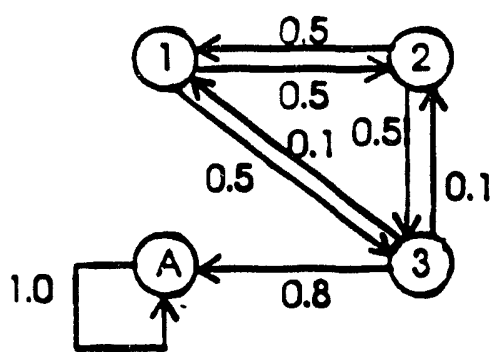

Figure 2.4 Network with absorbing state and transition probabilities

are the transition probabilities. For example, if the asset at node 1 is currently being inspected, there is a 0.5 probability that the asset at node 2 will be inspected next and a 0.5 probability that the asset at node 3 will be inspected next.

The data in the figure indicate that if asset 3 (the targeted asset) is being inspected, there is a 0.8 probability that the adversary will be detected and that the system will go to the state labeled " $A$ ", the absorbing state. If the system ever goes to state $A$, then it will remain in that state (the adversary has been captured). Of course the system must reach state $A$ iefore the adversary has had time to complete his tasks in order to effect capture.

\subsection{Markov Chain Model}

A Markov Chain Model (MCM) can be used to analyzed the surveillance problem for a given set of transition probabilities and target. The MCM uses the initial system state vector and the state transition matrix to compute the probability of adversary capture as a function of time. For a general discussion of MCMs, see Heyman and Sobel [1982]. 


\subsection{Model description}

The current state of the surveillance system can be computed using the following equation:

$$
x^{t}=P^{t} x^{0}
$$

where $\mathrm{x}^{\mathrm{t}}$, the $\mathrm{n} \times 1$ system state vector, contains the probabilities of finding the system in each of the $n$ states at time $t$. $P$ ? is the matrix of state transition probabilities raised to the power $t$, and $x^{0}$ is the state vector at time 0 .

One of the states in the state vector, $x^{t}$, corresponds to the absorbing state. It is desired to obtain the probability of system transition to the absorbing state as a function of time. After the first transition to the absorbing state, the system will remain in the absorbing state. Thus, an expression for the probability of a first transition to the absorbing state as a function of time is required. This probability of first transition from state $i$ to the absorbing state in period $t$ is denoted $\mathrm{f}_{\mathrm{i}}^{\mathrm{l}}$. A recursion relationship for $\mathrm{f}_{\mathrm{i}}^{\mathrm{l}}$ can be developed, as shown below.

$$
\begin{aligned}
& \mathrm{f}_{\mathrm{in}}^{l}=\mathrm{p}_{\mathrm{i}}^{1} \\
& f_{i s}^{2}=p_{i s}^{2}-f_{i s}^{1}=p_{i s}^{2}-p_{i s}^{1} \\
& f_{i 2}^{3}=p_{i s}^{3}-f_{i a}^{2}-f_{i s}^{1}=p_{i s}^{3}-p_{i a}^{2}+p_{i a}^{1}-p_{i s}^{2}=p_{i s}^{3}-p_{i s}^{2} \\
& f_{i s}^{4}=p_{i s}^{4}-p_{i s}^{3}
\end{aligned}
$$

where $\mathrm{p}_{\mathrm{s}}^{\mathrm{t}}$ is an element of the marrix $\mathrm{P}^{\mathrm{t}}$ (note that this is not the same value as the element $\mathrm{p}_{\mathrm{ij}}$ raised to the power $t$. The initial state vector can be multiplied by a vector of these first transition probabilities to determine the probability of absorption at time period $t$.

$$
\text { Prob(absorption at time } t)=f^{1} x^{0}=\sum_{i=1}^{n} f_{i=1}^{t} x_{i}^{0}
$$

\subsection{Transition probabilities, target selection, and game-theoretic implications}

The probability of detection will depend upon both the adversary's selection of a target and the transition probabilities specified in the design of the surveillance system. Given an assignment of transition probabilities, a rational adversary would choose to target the asset that corresponds to the minimum probability of detection.

The surveillance system design and target selection problems can be viewed as a two-stage game. In the first stage of the game, the surveillance system designer assigns the transition probabilities to arcs in the network. In the second stage of the game, the adversary 
observes these assigned probabilities and identifies the asset with the minimum detection probability. This minimum detection asset is then targeted by the adversary. Of course, the surveillance system designer should consider possible adversary responses when assigning transition probabilities to ares in the network.

Given a particular assignment of transition probabilities, the MCM can be used in an iterative manner to identify the most vulnerable target. Thus, the MCM can be used to solve the second stage of the game.

Solution of the complete two-stage game is beyond the scope of the model presented in this paper, but is discussed in the game theory literamure. Such games, often referred to as leader-follower or Stackelberg games, are discussed in (see Basar [1986], Edmunds and Bard [1991]). Algorithms for solving linear and a restricted class of nonlinear Stackelberg games are presented in these references.

\subsection{Computer Code and Example Application}

An interactive computer program has been developed to implement the procedures described above. The program allows the user to specify the elements of the state transition matrix and to identify the target node. The output of the program is the probability of first transition to the absorbing state as a function of time. The data input and analysis proceeds in accordance with the following steps.

Step 1 - Input dimension and elements of state transition matrix.

Step 2 - Input absorbing state.

Step 3 - Calculate elements of renormalized transition matrix.

Step 4 - Specify initial state vector. Set $t=1$ and $t_{\max }$.

Step 5 - Compute $\mathbf{P t}^{\mathbf{t}}$ and probability of first passage for time $t$.

Step 6 - Set $t=t+1$. Go to Step 5 if $t<t_{\max }$.

The renormalization in Step 3 is needed to account for the introduction of additional arcs leading from states to the absorption state. It is introduced for the convenience of the user. It allows the user to perform analyses with various target states without having to respecify the transition probabilities for the entire matrix, as in Step 1.

\subsection{Example problem}

The data input and calculations are illustrated with the following example. 
Step 1 - Input dimension and elements of state transition matrix.

The network diagram of the surveillance process is shown below. State transition probabilities are shown in the diagram. Note that the sum of the probabilities on all ares leaving each state is 1.0 .

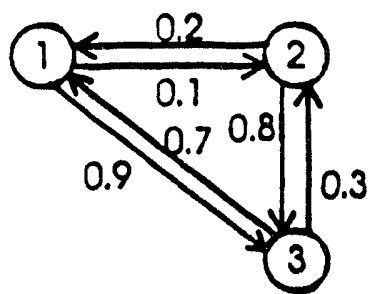

Figure 4.1 Network model of surveillance system states

Step 2 - Input absorbing state.

In the figure below, State 4 has been introduced as the absorbing state.

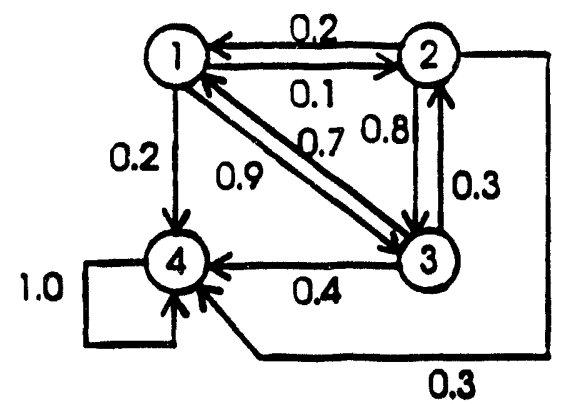

Figure 4.2 Network with absorbing state

Ares from each of the other states to the absorbing state have also been added. (This model addresses the more general case in which it is possible for the surveillance system to detect the adversary from more than one state.) Note that the sums of probabilities on ares leaving each state are greater than 1.0. The system now requires renormalization.

Step 3 - Calculate elements of renormalized transition matrix.

For each state, the transition probabilities for ares leaving the state are renormalized to 1.0. The results are shown below.

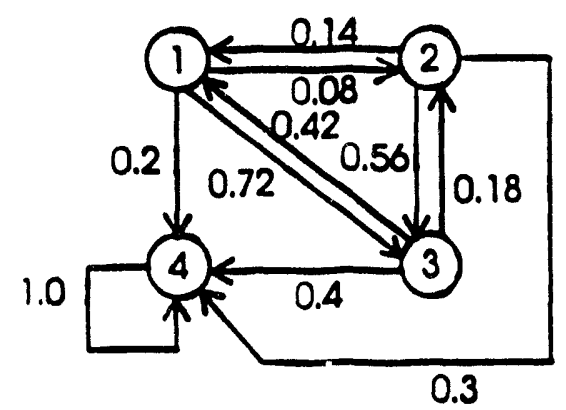

Figure 4.3 Network with renormalized transition probabilities 
The corresponding marrix of state transition probabilities is shown below.

$$
P=\left[\begin{array}{cccc}
0 & 0.08 & 0.72 & 0.2 \\
0.14 & 0 & 0.56 & 0.3 \\
0.42 & 0.18 & 0 & 0.4 \\
0 & 0 & 0 & 1
\end{array}\right]
$$

Step 4 - Specify initial state vector. Set $t=1$ and $t_{\max }$.

The program offers the user the options of two initial state vectors. First, the initial state of the system can be a uniform distribution over all possible states, including the absorption state. Second, the initial state of the system can be uniformily distributed over all states except the absorbing state. If the latter option is chosen, the inirial state vector is as shown below.

$$
\mathbf{x}^{0}=\left[\begin{array}{c}
0.333 \\
0.333 \\
0.333 \\
0
\end{array}\right]
$$

Step 5 - Compute $\mathrm{P}^{t}$ and probability of first passage for time $t$. The probability of first passage time for time $t=1$ is computed below.

$$
\mathrm{f}_{4}^{1}=\mathrm{x}^{0} \mathrm{P}^{1}(-, 4)=0.333(0.2)+0.333(0.3)+0.333(0.4)+0.333(1)=0.300
$$

where the notation $\mathrm{f}_{-\rightarrow}^{1}$ and $\mathrm{P}^{\mathrm{l}}(-, 4)$ refer to the transitions from all other states to state 4 , the absorbing state. This calculation indicates that the probability of the surveillance system detecting the adversary in one time step is 0.3 .

Step 6 - Set $t=t+1$. Go to Step 5 if $t<t_{\max }$.

The time index is changed to $t=2$, and the computations in Step 5 are repeated.

Step 5 - Compute $\mathrm{Pt}^{\mathrm{t}}$ and probability of first passage for time $\mathrm{t}$.

First, the matrix $\mathbf{P}^{2}$ is computed. The results of this computation are shown below. 


$$
\mathrm{P}^{2}=\left[\begin{array}{cccc}
0.314 & 0.130 & 0.045 & 0.512 \\
0.235 & 0.112 & 0.101 & 0.552 \\
0.025 & 0.034 & 0.403 & 0.538 \\
0 & 0 & 0 & 1
\end{array}\right]
$$

The probability of first passage time for time $t=2$ is computed below.

$$
\begin{aligned}
& f_{4}^{2}=x^{0}\left[P^{2}(-, 4)-P^{1}(-, 4)\right]= \\
& =0.333[0.512-0.2]+0.333[0.552-0.3]+0.333[0.538-0.4]+0=0.234
\end{aligned}
$$

This calculation indicates that the probability of the surveillance system detecting the adversary at the second time step is 0.234 .

If the theft, sabotage, or assault scenario requires two timesteps for completion, then the probability that the adversary is detected on either the first or the second timestep is equal to $0.534(0.3+0.234=0.534)$. If the adversary activity requires more than two time steps, then detection pmbabilities at later timesteps must also be calculated.

\subsection{Summary and Conclusions}

A Markov Chain Model has been developed to evaluate the effectiveness of randomized surveillance procedures. The model can be used to compute the probability that an adversary will be detected as a function of the time required for the adversary to complete his task. An interactive computer code has been written to perform the necessary computations. 


\section{References}

1) Heyman, Daniel P. and Matthew J. Sobel, Stochastic Models in Operations Research, Volume I: Stochastic Processes and Operating Characteristics, McGraw-Hill, New York (1982).

2) Basar, T., Dynamic Games and Applications in Economics, Springer-Verlag, New York (1986).

3) Edmunds, Thomas A. and Jonathan F. Bard, "Algorithms for Nonlinear Bilevel Mathematical Programs," IEEE Transactions on Systems, Man, and Cybernetics, Vol. 21, No. 1, (1991). 
11
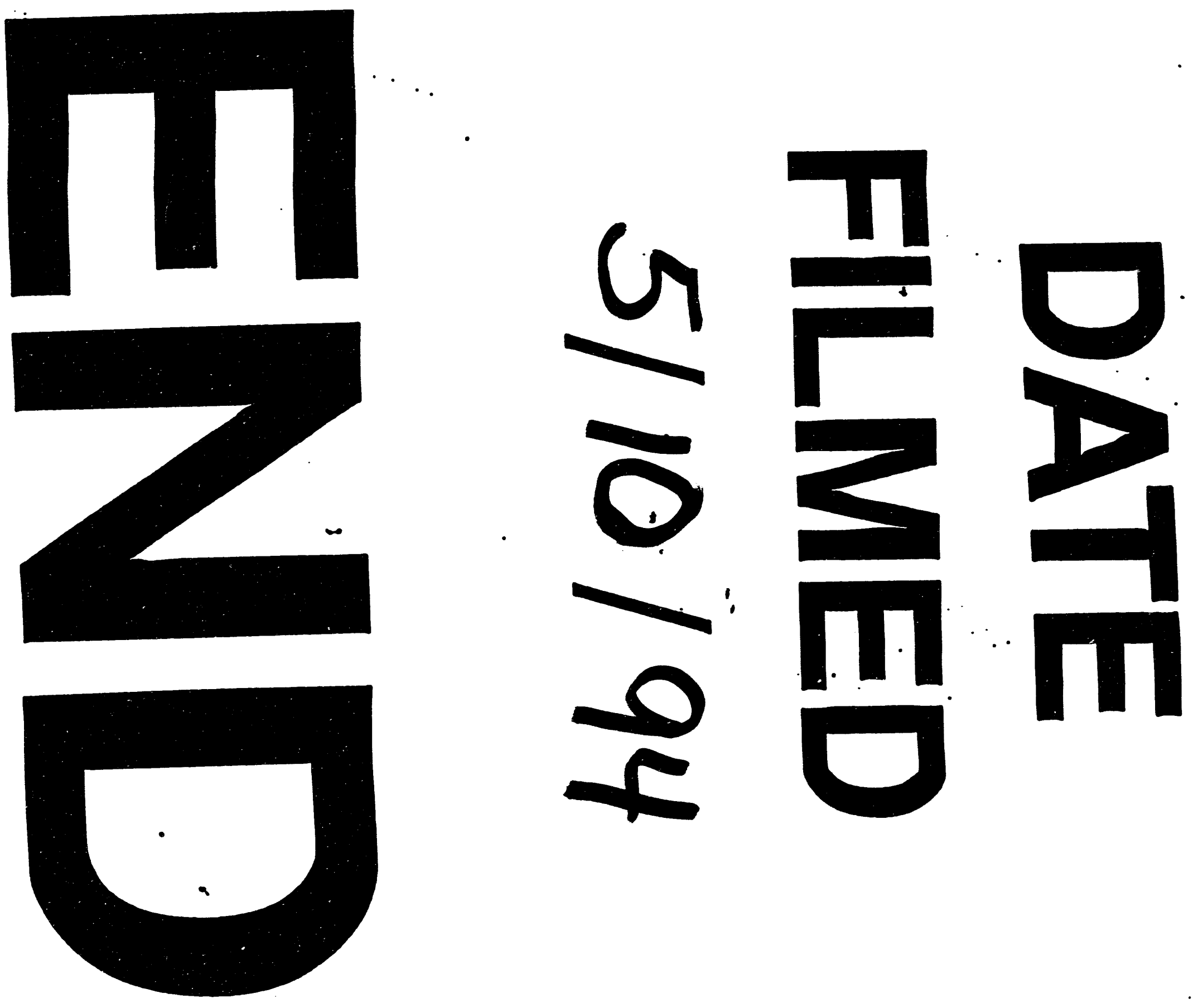

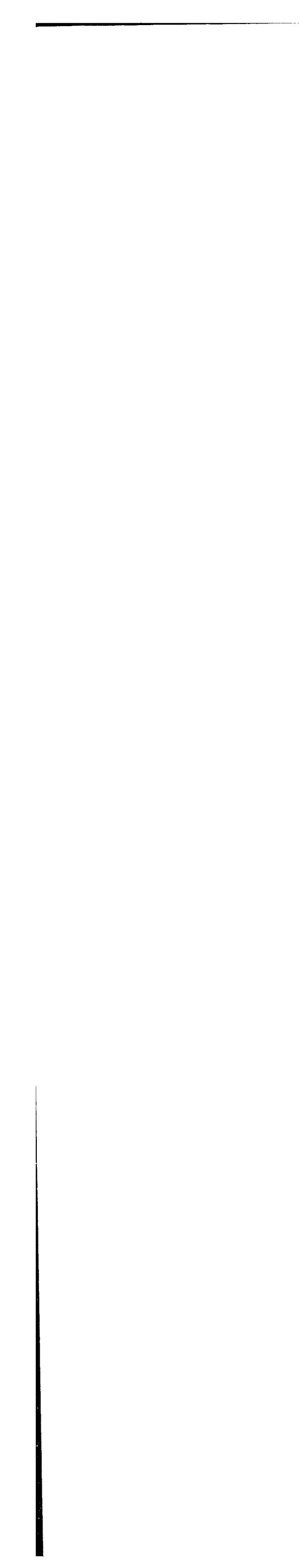